\title{
Materias primas críticas y complejidad económica en América Latina
}

\author{
Critical Raw Materials and Economic \\ Complexity in Latin America
}

\section{Matérias-primas críticas e complexidade econômica na América Latina}

\author{
Juan Sebastián Lara-Rodríguez * \\ André Tosi Furtado \\ Aleix Altimiras-Martin ${ }^{* * *}$
}

DOI: https://doi.org/10.19053/01203053.v37.n65.2018.5426

Fecha de recepción: 19 de septiembre de 2016

Fecha de aprobación: 18 de septiembre de 2017

\footnotetext{
* Magíster en Política Científica y Tecnológica. Asistente de investigación. Departamento de Política Científica e Tecnológica, Universidade Estadual de Campinas, CEP: 13083-870, Estado de São Paulo, Brasil. Correo electrónico: juselaro@gmail.com - iD http://orcid.org/0000-0003-4795-754X

** Doctor en Economía del Espacio y el Medio Ambiente, Université Paris I Pantheón Sorbonne. Posdoctorado, Centre de Recherche Sur L'environnment et le Développement. Profesor titular. Departamento de Política Científica e Tecnológica, Instituto de Geociências, Universidade Estadual de Campinas, CEP: 13083-870, Estado de São Paulo, Brasil. E-mail: furtado@ige.unicamp.br - (D) http://orcid.org/0000-0002-6183-192X

*** Doctor en Economía Agrícola, University of Cambridge. Profesor asociado. Departamento de Política Científica e Tecnológica, Instituto de Geociências, Universidade Estadual de Campinas, CEP: 13083-870, Estado de São Paulo, Brasil. E-mail: aleix@ige.unicamp.br Scopus ID: 56340047200
} 


\section{Resumen}

Existen minerales dinamizadores de crecimiento económico, fundamentales para el desarrollo de tecnologías sostenibles. Estas materias primas críticas (MPC) son determinadas por modelos creados para economías complejas. El objetivo de este artículo es examinar las políticas minerales de materias primas críticas en las principales economías de América Latina, y el papel de sus respectivos sistemas nacionales de innovación (SNI), en búsqueda de mayor complejidad económica, mediante un método de evaluación comparativo aplicado a la política mineral de las principales naciones de esta zona - Brasil, México, Argentina, Colombia y Chile- Descubrimos que debido a la simplicidad de estas economías y de políticas minerales que desestiman sus respectivos sistemas nacionales de innovación, se compromete el aumento de la complejidad económica de los Estados en cuestión, la cual se caracteriza por la precaria adición de valor mediante la interacción de conocimiento y capacidades en relación con sus recursos minerales e industria.

Palabras clave: recursos minerales, recursos naturales no renovables, desarrollo económico, innovación, tecnologías sostenibles.

Clasificación JEL: Q32; O30; N56; L72; Q55 


\section{Abstract}

There are minerals that boost economic growth and which are essential for the development of sustainable technologies. These critical raw materials (CRMs) were determined by models created for complex economies. This paper aims to examine the mineral policies regarding CRMs of the main Latin-American economies, and the role of their respective National Innovation Systems (NIS) in the pursuit of greater economic complexity. This is achieved through a comparative assessment method applied to the mineral policies of the principal nations of the region - Brazil, Mexico, Argentina, Colombia and Chile. In this way, we found that due to the simplicity of these economies, as well as mineral policies that disregard their respective NIS, the increase of the economic complexity of the states in question is compromised. This is characterized by the exiguous value added through the interaction of knowledge and capabilities regarding their mineral resources and industry.

Keywords: mineral resources, non-renewable resources, economic development, innovation, sustainable technologies. 


\section{Resumo}

Existem minérios que impulsam o crescimento econômico e são fundamentais para o desenvolvimento de tecnologias sustentáveis, estas Matérias Primas Criticas (MPC) são determinadas mediante modelos criados pelas economias complexas. Portanto, o artigo tem por objetivo examinar as políticas minerais de MPC nas economias Latino Americanas de destaque, e o papel do respetivo Sistema Nacional de Inovação (SNI) na procura de uma maior complexidade econômica. Isto, por meio de um método de avaliação comparativa de política mineral, aplicado nos principais países da América Latina -Brasil, México, Argentina, Colômbia e Chile-. Desta maneira a gente descobriu que devido à simplicidade destas economias, e de políticas minerais que desestimam seus SNI, há um comprometimento do aumento da complexidade econômica dos países estudados. A qual é caraterizada pela precária adição de valor através da interação do conhecimento e capacidades, em torno dos recursos minerais e a indústria.

Palavras-chave: Recursos minerais; recursos naturais não renováveis; desenvolvimento econômico; inovação; tecnologias sustentáveis. 


\section{INTRODUCCIÓN}

En la actualidad existe una especial atención sobre el desarrollo sostenible ${ }^{1}$ $\mathrm{y}$ una preocupación generalizada por el impacto antrópico en el ambiente. Impacto que se manifiesta en cambios (científicamente constatados) drásticos y difícilmente reversibles en el sistema global, y de los cuales citamos algunos ejemplos: el cambio climático y el calentamiento global originados por la producción de gases de efecto invernadero, la población humana cercana a 10 billones de personas para el año 2050, la deforestación, la acidificación de los mares, la plastificación de los ecosistemas, los incrementos de los niveles de consumo, el desgaste virtual de la actualización u obsolescencia programada de tecnologías innovadoras, todos los cuales generan desechos inorgánicos en magnitudes incontrolables.

En la investigación para disminuir los impactos ambientales y contribuir al avance de los cambios tecnológicos. Así como en la búsqueda de formas de generar energía de manera sostenible e impulsar tecnologías de consumo innovadoras, entre otras aplicaciones de innegable importancia social para las naciones. Se advierte la necesidad de

\footnotetext{
1 El concepto de desarrollo sostenible más reconocido es el contenido en el Reporte Brundtland, catalogándolo como la situación donde se "satisfacen las necesidades del presente sin comprometer la capacidad de las generaciones futuras de satisfacer sus propias necesidades" (World Commission on Environment and Development, 1987, p. 16). En torno a la discusión sobre desarrollo sostenible y su relación significativa con el crecimiento económico y los recursos naturales véanse Auty (2003, 2007), Hartwick (1977), Karl (1997), Van der Ploeg (2011).
} 
una serie de materiales, principalmente minerales, escasos y de riesgoso abastecimiento, que son estratégicamente relevantes para el funcionamiento de las denominadas economías avanzadas, así como de aquellas que están en el proceso de implantar una sociedad basada en el aprendizaje, las cuales, conjuntamente, son o pueden llegar a ser productores de estos recursos no renovables, también denominados como materias primas críticas.

Una forma interesante de diferenciar las economías avanzadas de aquellas que se encuentran en el proceso de implantar una sociedad basada en el aprendizaje, es su complejidad económica ${ }^{2}$. Para Hidalgo y Hausmann (2009), la complejidad económica parte de la relación entre la riqueza y el desarrollo; esa complejidad son las interacciones entre un número creciente de actividades individuales que conforman una economía; por lo tanto, la productividad de un país reside en la diversidad de sus "capacidades" no comerciables, de manera que las diferencias de ingreso entre países pueden ser explicadas por la diferencia de complejidad económica, la cual es medida mediante la diversidad de capacidades presentes en un país y sus interacciones en red.

El método de reflexiones desarrollado por Hidalgo y Hausmann (2009) produce un conjunto simétrico de variables de dos tipos de nodos para capturar la estructura de la red: los niveles de diversificación de un país (número de productos exportados por el país o con varios niveles de especialización), y la ubicuidad del producto (el número de países que exportan el producto y cuya ventaja comparativa es relevada).

Los académicos mencionados reconocen que la complejidad económica no enfatiza el proceso por el cual los países acumulan capacidades o conocimiento, sino que se enfoca en su medición y consecuencias. Ante este vacío, la economía evolutiva ${ }^{3}$ es propuesta como una alternativa dialógica. Esta se cimienta en un marco de conceptos schumpeterianos y neoschumpeterianos, que son provenientes de una metáfora evolutiva biológica, relacionada esencialmente con la variación, la selección y la retención de atributos (Lara-Rodríguez, Rojas \& Martínez, 2015). Esta corriente teórica económica trata una serie de fenómenos sociales que son de limitada

\footnotetext{
2 El concepto de complejidad económica puede corresponder a discusiones epistemológicas planteadas en las ciencias económicas, como las presentadas por Koppl (2010) sobre el posicionamiento de una economía representada en literatura o matemática, simultáneamente, y la retroacción de la teoría de la complejidad en la teoría económica.

3 Para conocer los trabajos académicos fundamentales de la corriente económica evolutiva véanse Freeman y Soete (1997), Nelson y Winter (1982), Dosi (1982).
} 
respuesta por parte del marco de la teoría económica neoclásica, como por ejemplo, la explicación del mayor comportamiento económico humano del emprendimiento y la innovación -destrucción creativa-, los cuales, indudablemente, son elementos vigorizantes del crecimiento y el desarro1lo. Y el sistema de innovación, por su parte, es una vertiente de investigación de esta corriente, la cual básicamente se enfoca en los flujos de tecnología e información entre las personas, las empresas y las instituciones, las cuales son protagonistas clave del proceso innovador, y estos flujos, a su vez, deben ser dinamizados mediante una política de innovación (Lara-Rodríguez \& Bermúdez, 2011).

Los sistemas de innovación cuentan con una partición particular que depende del ámbito (nacional, regional, sectorial, tecnológico, corporativo, etc.). Lundvall, Vang, Chaminade y Chaminade (2009) definen el sistema nacional de innovación (SNI) como un sistema abierto, evolutivo y complejo, que incluye relaciones dentro y entre organizaciones, instituciones y estructuras socioeconómicas, que determinan la tasa y la dirección de la innovación y la construcción de competencia, emanada de la ciencia y del aprendizaje basado en experiencia. Las políticas públicas en este contexto deben ser creadas de tal manera que consigan buenas estructuras e instituciones, por tanto, el aprendizaje tecnológico es fundamental; pero, solo a partir del aprendizaje social y político se pueden construir instituciones y estrategias políticas que soporten la construcción de competencia.

Actualmente, en América Latina no hay estudios que eluciden con certeza de qué manera se determinan las materias primas críticas en las economías complejas, cuáles son sus aplicaciones tecnológicas o qué productos se fabrican con ellas, y quiénes son sus principales oferentes. Igualmente, es necesario determinar si las políticas minerales de esta región están destinadas a fijar las materias primas críticas para sus economías, y si estas políticas incorporan su respectivo sistema nacional de innovación en búsqueda de mayor complejidad económica. Por tanto, el artículo tiene por objetivo central examinar las políticas minerales de materias primas críticas en las principales economías de América latina, y el papel de su respectivo sistema nacional de innovación, en búsqueda de mayor complejidad económica. Además, la hipótesis consiste en constatar si, según la complejidad económica de una nación, se establece la variedad de sus materias primas críticas.

En la siguiente sección se conceptualizan las materias primas críticas, dilucidando modelos, criterios para su determinación y resultados en las economías más complejas del 
mundo; en la sección 3 se presentan unas consideraciones sobre las materias primas críticas, se definen las aplicaciones tecnológicas o productos complejos fabricados con estos elementos, que en parte son fundamentales para inhibir externalidades negativas ambientales de actividades antrópicas; en la sección 4 se exponen generalidades acerca de la oferta de estos minerales a nivel mundial, concretamente y con una visión geopolítica se trata el tema de los monopolios de extracción y reservas; en la sección 5 se estudia la complejidad de las principales economías de América Latina, así como las materias primas relevantes producidas por estas y su distribución, y se presenta una descripción de las directrices de política mineral dirigidas a la determinación de las materias primas críticas en estas naciones; y en la sección 6 se plantea una discusión sobre las políticas minerales de las principales economías de América Latina, argumentando que estas no conducen a una mayor complejidad económica, con base en la desestimación de los diferentes sistemas nacionales de innovación para la formulación e implementación de políticas minerales.

\section{CONCEPTUALIZACIÓN DE LAS MATERIAS PRIMAS CRÍTICAS}

La escasez o la abundancia de recursos naturales no renovables son dilemas en que históricamente se han basado estudios de varias disciplinas de las ciencias básicas y aplicadas, tanto en áreas sociales como naturales. El debate genérico originado, continúa un proceso evolutivo, en el cual emergen nuevas y cada vez más singulares adaptaciones teórico-conceptuales, con el objeto de explicar la naturaleza de la distribución. Desde esta noción se reconoce la necesidad de las poblaciones humanas de satisfacer sus demandas de consumo y, por tanto, de acceder a ciertos recursos naturales materias primas - para desarrollar actividades económicas que mantengan o incrementen sus niveles subjetivos de bienestar.

Según Chakhmouradian, Smith y Kynicky (2015), durante la Primera Guerra Mundial, cuando las naciones involucradas en el conflicto bélico comenzaron a experimentar severos recortes de los materiales requeridos para mantener el esfuerzo de guerra, cuando Alemania aseguró el control de la producción de tungsteno y las demás potencias debieron luchar por fuentes alternativas, los políticos, economistas y empresarios emplearon el término 'criticalidad'.

El término criticalidad fue utilizado por el Comité de Asuntos Interiores e Insulares del Senado de los Estados Unidos (1954), el cual, mediante resolución, investigó el acceso a doce materiales, aparte de hidrocarburos. 
En la actualidad, los minerales catalogados como avanzados o críticos, también son conocidos como metales tecnológicos, metales verdes o raros, o también materias primas críticas, los cuales son principalmente divulgados desde instituciones gubernamentales, que tienen por objeto la prospectiva económica de los Estados con una importante participación de sus sistemas nacionales de ciencia, tecnología e innovación.

En el caso de los Estados Unidos de América, el National Research Council (2008), por medio del Committee on Critical Mineral Impacts on the U.S. Economy, establece la importancia de algunos minerales no combustibles para su sociedad. En el informe, las dos dimensiones más importantes de criticalidad son: (1) importancia de uso, desde la idea de que algunos minerales no combustibles son más importantes en su uso que otros, y su sustitución se convierte en concepto clave, puesto que el remplazo puede ser muy costoso o técnicamente dificultoso; y (2) disponibilidad, la cual parte del proceso minero y refleja un número de consideraciones a medio o largo plazo (v.g., geológicas, técnicas, ambientales, sociales, políticas y económicas). Paralelamente determina una matriz de criticalidad (Figura 1), cuya metodología utiliza una escala relativa de 1 (bajo) a 4 (alto); el eje vertical tiene en cuenta indicadores de consumo de los EUA, porcentajes de consumo de este país en usos existentes, en los cuales la sustitución es difícil o imposible, y usos emergentes que alteren la tasa de producción global. El eje horizontal se remite a indicadores de reservas mundiales, dependencia de importaciones de los EUA, producción mundial por producto como porcentaje de la producción primaria, y producción secundaria de los EUA como su consumo. Por ejemplo, en la Figura 1, el rodio es más crítico que el cobre.

Otra apreciación que cataloga a varios minerales y los denomina como materias primas criticas (MPC) proviene de la European Commission (2014), la cual describe estas materias primas como fundamentales para la economía de la Unión Europea (UE), el crecimiento y el empleo, además de ser básicas en el mejoramiento de la calidad de vida, e igualmente se condicionan a un alto riesgo de suministro. Estas materias primas no energéticas están intrínsecamente vinculadas a todas las industrias a lo largo de los niveles de las cadenas de abastecimiento, consecuentemente, son esenciales para la vida común, ya que gran cantidad de productos están fabricados con estos materiales. 

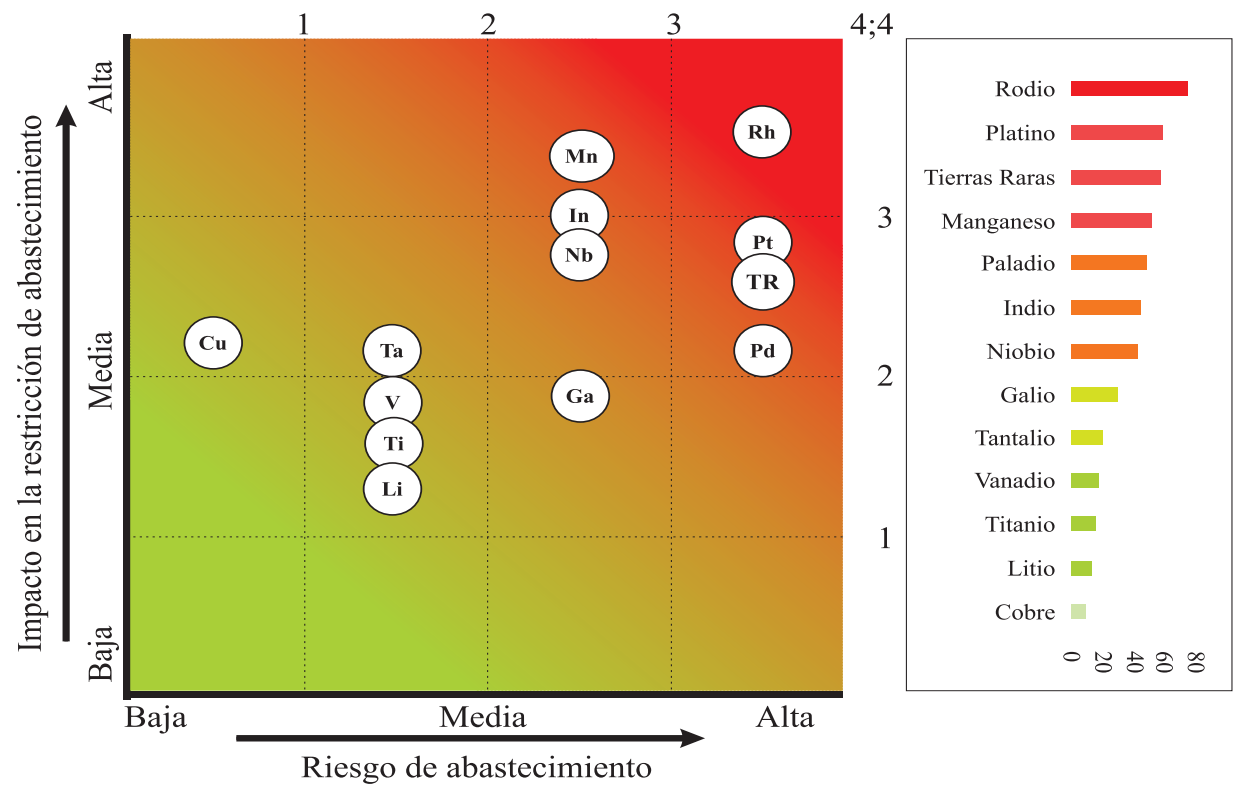

Figura 1. Matriz de criticalidad de minerales EUA.

Fuente: adaptación a partir del National Research Council of the National Academies (2008); Glöser, Tercero, Gandenberger y Faulstich (2015).

Igualmente existen otros estudios metodológicos relacionados con la identificación de estos materiales, que son analizados por Erdmann y Graedel (2011), como los adelantados por el Departamento de Energía de los Estados Unidos (DOE); la General Electric; el Instituto de Análisis para la Defensa de los Estados Unidos; la Asociación de Empresas de Baviera IW Consult; El NEDO de la Mitsubishi Research and Consulting; el Oakedene Hollins Study; el programa ambiental de las Naciones Unidas del Öko-Institute y el Instituto Fraunho- fer para sistemas de innovación. Otro avance metodológico destacable es el propuesto por Graedel et al. (2012), quienes llevan la criticalidad del material a tres niveles organizacionales (v.g., corporativo, nacional y global), incorporando información de una dispar variedad de especialidades y fuentes de datos, desde la geología, la tecnología, la economía, el comportamiento humano, las evaluaciones de expertos, etc., y colecta datos tanto cuantitativos como cualitativos, unos bien definidos y otros menos. 
Las materias primas críticas son catalogadas, como se expone en la Figura 2 , en el eje horizontal por importancia económica, resultado de la evaluación de la proporción de materiales asociados a megasectores industriales. Estas proporciones son combinadas con el valor agregado bruto (VAB) de los megasectores al producto interno bruto (PIB) de la UE, y este total se compara de acuerdo con el PIB total del bloque económico. El eje vertical riesgo de abastecimiento está compuesto por tres factores: (1) índice de sustituibilidad, que mide la dificultad de sustituir el material calificado medido en todas las aplicaciones; (2) tasas de reciclaje fin-ciclo-de-vida, las cuales miden la proporción de metal y productos fabricados a partir de chatarra $\mathrm{y}$ otros productos que contengan metal con baja tasa de residuos, y (3) factores de concentración, que parten del índice Herfindahl-Hirschman, modificado con el indicador de gobernanza del World Governance Index (WGI). Por tanto, en total se contemplan 54 materias primas, de las cuales las 20 situadas en el campo más claro de la Figura 2 corresponden a críticas.

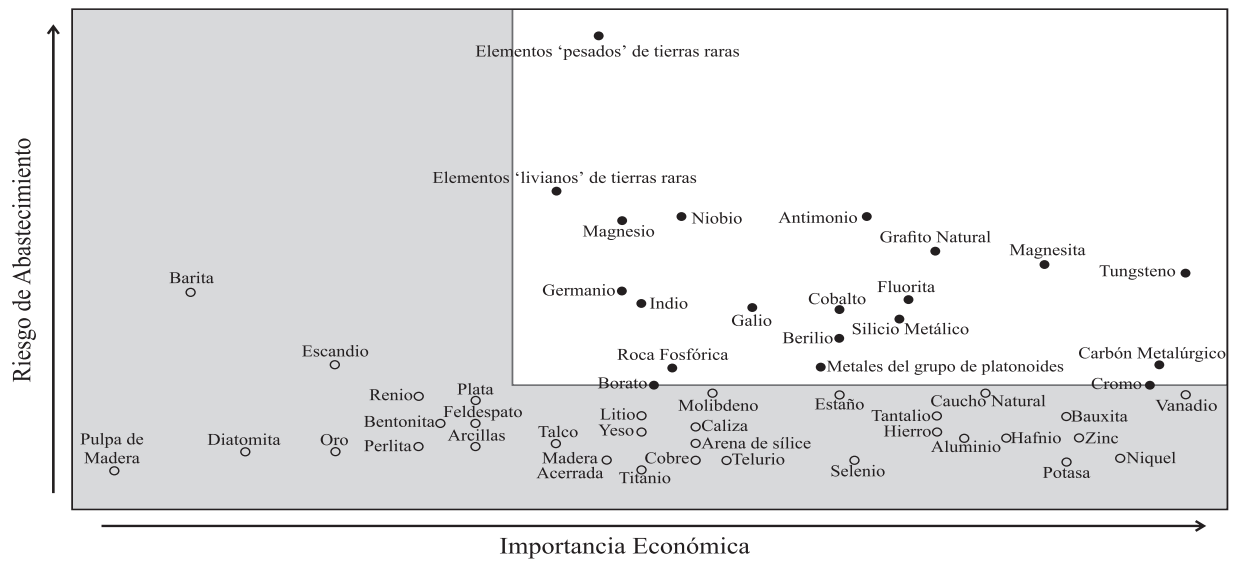

Figura 2. Materias primas críticas en la Unión Europea.

Fuente: adaptación a partir de la European Commission (2014).

El término materias primas críticas cuenta con varias interpretaciones como metodologías para su determinación. Sievers, Buijs y Tercero
Espinoza (2012) sintéticamente las definen como minerales no energéticos, que coordinan una relativa alta importancia económica, comparativamente 
con el alto riesgo de interrupciones en su suministro. Además se debe mencionar que la unicidad de criterios cualitativos e indicadores para el establecimiento de estos elementos, debido a las variaciones de ambiente evidentes, continúa una adaptación singular dependiendo de la complejidad económica de los mercados que demandan estos recursos.

En lo relacionado con la complejidad económica, Hausmann et al. (2014) la expresan en la composición de la productividad del país y refleja las estructuras de mantener y combinar el conocimiento en forma de redes. Estos autores señalan que las economías complejas tienen enormes cantidades de capacidades relevantes juntas, a través de amplias redes de personas, para generar una diversa mezcla de productos intensivos en conocimiento; en contraste, las economías simples tienen una estrecha base de capacidades productivas, lo que las lleva a producir bienes simples con bajas interacciones en red. El indicador que las intenta cuantificar es denominado índice de complejidad económica (ICE) (Hausman et al., 2014), el cual surge de las nociones de diversidad y ubicuidad de productos menos y más complejos entre países.

Por otro lado, la intensidad en investigación y desarrollo (I+D) representa el porcentaje del PIB dirigido a actividades de ciencia, tecnología e innovación e impulso al sistema nacional de innovación. Este se encuentra proporcionalmente vinculado a una mayor complejidad económica. Como se retrata en la Tabla 1, son determinados los primeros productos internos brutos del mundo, siendo mayor el de la UE, seguido por el de los EUA. Además, se dilucidan datos de las principales economías de los Estados miembros del bloque económico europeo, para demostrar fluctuación en los indicadores entre los 28 Estados miembros. La Tabla 1 propone justificar la escogencia y el protagonismo de las materias primas críticas catalogadas en estas economías, en razón de su liderazgo productivo global. El índice de complejidad económica de la Unión Europea se calculó de acuerdo con el promedio de los Estados miembros presentes en el Atlas de Complejidad Económica. Malta, Luxemburgo y Chipre fueron excluidos, debido a la ausencia de sus índices, y se soslaya el Brexit, puesto que el modelo fue diseñado en el 2014. Adicionalmente, los datos de intensidad en I+D fueron tomados según la ponderación más reciente del Banco Mundial, en el caso de la Unión Europea las cifras son del 2013, y para los EUA del año 2012. 
Tabla 1. Productividad, I+D y complejidad económica de la UE y los EUA.

\begin{tabular}{|c|c|c|c|}
\hline & PIB en USD & I+ & ICE $^{\mathbf{b}}$ \\
\hline Unión Europea & $18.510 .000 .000 .000,00$ & $2,02 \%$ & 1,134 \\
\hline Alemania & $3.868 .291 .231 .823,77$ & $2,85 \%$ & 1,922 \\
\hline Reino Unido & $2.988 .893 .283 .565,20$ & $1,63 \%$ & 1,481 \\
\hline Francia & $2.829 .192 .039 .171,84$ & $2,23 \%$ & 1,291 \\
\hline Italia & $2.141 .161 .325 .367,43$ & $1,26 \%$ & 1,356 \\
\hline España & $1.381 .342 .101 .735,68$ & $1,24 \%$ & 0,824 \\
\hline Estados Unidos & $17.410 .000 .000 .000,00$ & $2,81 \%$ & 1,357 \\
\hline
\end{tabular}

Notas: a(The World Bank, 2014) ${ }^{\mathrm{b}}$ (The World Bank, 2013) c(Hausmann et al., 2014).

CONSIDERACIONES DE LA DEMANDA POR MATERIAS PRIMAS CRÍTICAS

Actualmente, las aplicaciones de las materias primas críticas están concentradas principalmente en el campo de las altas tecnologías, específicamente utilizadas en componentes avanzados, como celdas de combustible, pantallas de teléfonos móviles, baterías de alta capacidad de almacenamiento, magnetos permanentes para aerogeneradores, dispositivos de energía verde, diodos emisores de luz (LED), tecnologías bajas en carbono, fibra óptica, circuitos integrados, discos duros, refinamiento de petróleo, paneles fotovoltaicos, convertidores catalíticos, láseres, aplicaciones de defensa, aleaciones de metalurgia avanzada, polvos de abrasión, aditivos de vidrios, fósforos, catalizadores para automóviles eléctricos, fluido catalizador de agrietamiento, superconductores, aplicaciones médicas y aeroespaciales, entre otras (Busch, Steinberger, Dawson, Purnell \& Roelich, 2014; Du \& Graedel, 2013; Hensel, 2011; Massari \& Ruberti, 2013).

La incerteza de contar con proveedores estables de materias primas críticas lleva a la exploración de alternativas tecnológicas y científicas ambiciosas. Por ejemplo, la sustitución mediante la utilización de nanotecnología descrita por Fromer y Diallo (2013), simultáneamente la búsqueda de materiales substitutos y mayor disponibilidad hecha por Halme, Piirainen, Vekinis, Ernst-Udo y Viljamaa (2012). Sin embargo, en estos trabajos se determinan bajas tasas de rendimiento y fiabilidad de los sustitutos, altas inversiones de capital para $\mathrm{I}+\mathrm{D}$, con largos periodos para resultados constantes. Además 
del incremento de los costos de mantenimiento de las aplicaciones tecnológicas, y sin olvidar la identificación de bajas tasas de reciclaje o disipación de los materiales en el ambiente, desde que son extraídos, convertidos, utilizados y deshechos (Zimmermann \& Gößling-Reisemann, 2013). Otra alternativa para satisfacer la escasez es la minería en aguas oceánicas profundas, que produce altos riesgos ambientales y poca certeza, debido a la falta de exploración de nódulos oceánicos (Hein, Mizell, Koschinsky \& Conrad, 2013).

Con relación a sistemas tecnológicos, la determinación de las materias primas críticas también se hace visible. En el caso de la energía solar fotovoltaica, Goe y Gaustad (2014) determinan, según sus métricas para los EUA, que el platino, germanio, telurio, indio, arsénico, silicio, estaño, selenio, molibdeno, plata, cadmio, zinc, galio, oro, aluminio, hierro y cobre, son en su orden los más críticos. Para otro sistema tecnológico de energía renovable como lo es el eólico, Brumme (2014) menciona que los elementos cruciales para el sistema son principalmente las tierras raras, que son utilizadas en los magnetos permanentes de los aerogeneradores, y tienen una intensidad de 0.2 ton/MW, lo cual indica $71.25 \%$ de neodimio, o sea $145.5 \mathrm{~kg} / \mathrm{MW} ; 25$ $\%$ de praseodimio $50 \mathrm{~kg} / \mathrm{MW} ; 3.75$ $\%$ de disprosio equivalente a $7.5 \mathrm{~kg} /$ MW. Otro sistema tecnológico es el de la movilidad, desplegado en la Tabla 2 , en donde se mencionan las tecnologías y los elementos estratégicos para su desarrollo.

Tabla 2. Movilidad baja emisora de contaminantes y sus MPC

\begin{tabular}{|l|c|c|c|c|c|c|c|c|}
\hline Tecnología & Cobre & Cobalto & Plomo & Litio & $\begin{array}{c}\text { Man- } \\
\text { ganeso }\end{array}$ & Niobio & MGP a & ETR \\
\hline Vehículo de gas natural & $\mathrm{X}$ & & $\mathrm{X}$ & & & $\mathrm{X}$ & $\mathrm{X}$ & \\
\hline $\begin{array}{l}\text { Vehículo celda de } \\
\text { combustible }\end{array}$ & $\mathrm{X}$ & & $\mathrm{X}$ & & & & $\mathrm{X}$ & \\
\hline Vehículo híbrido eléctrico & $\mathrm{X}$ & & & & & $\mathrm{X}$ & $\mathrm{X}$ & $\mathrm{X}$ \\
\hline $\begin{array}{l}\text { Vehículo híbrido } \\
\text { eléctrico de enchufe }\end{array}$ & $\mathrm{X}$ & $\mathrm{X}$ & & $\mathrm{X}$ & $\mathrm{X}$ & & $\mathrm{X}$ & \\
\hline $\begin{array}{l}\text { Vehículo eléctrico } \\
\text { completo }\end{array}$ & $\mathrm{X}$ & $\mathrm{X}$ & & $\mathrm{X}$ & $\mathrm{X}$ & & & \\
\hline Motor eléctrico & $\mathrm{X}$ & & & & & & & $\mathrm{X}$ \\
\hline
\end{tabular}

Nota: ${ }^{a}$ MGP: metales del grupo de los platinoides; ETR: elementos de tierras raras.

Fuente: adaptación a partir de Ziemann, Grunwald, Schebek, Müller y Weil, (2013). 
La discriminación de las principales aplicaciones tecnológicas de los elementos de tierras raras (ETR), ocho livianos y ocho pesados, es ampliada en la Tabla 3; la de los seis metales del grupo del platino (MGP) se expone en la Tabla 4, junto con las demás materias primas críticas catalogadas. Lo cual nos da una idea de la diversidad de aplicaciones tecnocientíficas que, una vez difundidas en el mercado, se traducen en productos complejos.

Tabla 3. Aplicaciones tecnológicas de los elementos de tierras raras

\begin{tabular}{|l|l|}
\hline Elemento & \multicolumn{1}{c|}{ Aplicaciones tecnológicas } \\
\hline Lantano & $\begin{array}{l}\text { Material de más del } 50 \text { \% de los lentes de cámaras digitales, incluyendo las de te- } \\
\text { léfonos. La catálisis basada en La es usada para refinar petróleo. Baterías hibridas } \\
\text { de metal-níquel son construidas con ánodos y aleaciones de La (vehículos híbridos } \\
\text { eléctricos), Mischmetal (Van Gosen et al., 2014) }\end{array}$ \\
\hline Cerio & $\begin{array}{l}\text { La catálisis es utilizada en convertidores catalíticos automovilísticos, Mischmetal; } \\
\text { catálisis, aleaciones metálicas, blindaje radioactivo, fósforos para pantallas delgadas } \\
\text { (Van Gosen et al., 2014). }\end{array}$ \\
\hline Praseodimio & $\begin{array}{l}\text { Vidrio de seguridad para soldadores, Mischmetal, magnetos utilizados en una varie- } \\
\text { dad convencional de subsistemas de carros: dirección asistida, ventanas eléctricas, } \\
\text { asientos eléctricos, altoparlantes, tecnologías para la generación de energía limpia, } \\
\text { televisores de pantalla delgada, mini-motores eléctricos portables, discos duros, } \\
\text { magnetos, láseres, refrigeradores criogénicos (Slowinski, Latimer \& Mehlman, 2013; } \\
\text { Van Gosen et al., 2014). }\end{array}$ \\
\hline Neodimio & $\begin{array}{l}\text { Aleaciones en magnetos permanentes, imanes utilizados en los discos duros de } \\
\text { computadores y reproductores de CD y DVD, Discos duros, aplicaciones médicas, } \\
\text { minimotores eléctricos portables, magnetos permanentes de alta resistencia. Los ae- } \\
\text { rogeneradores pueden contener 1 tonelada de neodimio por megawatt de capacidad } \\
\text { eléctrica generada (Slowinski et al., 2013; Van Gosen et al., 2014) (1; Hein et al., } \\
\text { 2013; Goonan, 2011). }\end{array}$ \\
\hline Prometio & $\begin{array}{l}\text { Natural no existe en la tierra, pintura luminosa, baterías nucleares, y tiene posibles fu- } \\
\text { turas aplicaciones como fuente portable de rayos X, y su calor auxiliar puede proveer } \\
\text { fuente energética para pruebas espaciales y satélites (Gupta, Jain, Hamdan, Agarwal } \\
\text { \& Bharti, 2010). }\end{array}$ \\
\hline
\end{tabular}


Materias primas críticas y complejidad económica en América Latina

Juan Sebastián Lara-Rodríguez - André Tosi Furtado - Aleix Altimiras-Martin

Continuación de la Tabla 3.

\begin{tabular}{|c|c|c|}
\hline Samario & \multicolumn{2}{|c|}{$\begin{array}{l}\text { Imanes para defensa (v.g., referenciamento y navegación, radares, motores de mag- } \\
\text { netos permanentes) subsistemas de automóviles: dirección asistida, ventanas eléctri- } \\
\text { cas, asientos eléctricos, y parlantes, tecnologías para la generación de energía limpia } \\
\text { (Hensel, 2011; Van Gosen et al., 2014). }\end{array}$} \\
\hline Europio & \multicolumn{2}{|c|}{$\begin{array}{l}\text { Fósforos utilizados en tubos de rayos catódicos, lámparas fluorescentes, y otras apli- } \\
\text { caciones que requieren color en la luz exhibida (Goonan, 2011). }\end{array}$} \\
\hline Gadolinio & \multicolumn{2}{|c|}{$\begin{array}{l}\text { Agente de contraste para imágenes de resonancia magnética, circuitos integrados de } \\
\text { memoria, fósforos (Hein et al., 2013; Goonan, 2011). }\end{array}$} \\
\hline Terbio & \multicolumn{2}{|c|}{$\begin{array}{l}\text { Luces rojas-verdes-azules, fósforos utilizados en varias lámparas, paneles, televisores } \\
\text { y aplicaciones médicas (Van Gosen et al., 2014). }\end{array}$} \\
\hline Disprosio & \multicolumn{2}{|c|}{ Imanes y motores utilizados en automóviles eléctricos (Goonan, 2011). } \\
\hline Holmio & \multicolumn{2}{|c|}{$\begin{array}{l}\text { Láseres en estado sólido, utilizados en endoscopias y en la atmosfera, como en la } \\
\text { aviación, para el registro de tormentas y eventos cercanos a las torres de control, } \\
\text { parte estándar de los sistemas de navegación de aviación comercial (Abramczyk, } \\
\text { 2005). }\end{array}$} \\
\hline Erbio & \multicolumn{2}{|c|}{$\begin{array}{l}\text { Amplificadores de erbio-dopados para fibra óptica. Los láseres son utilizados para } \\
\text { cortar huesos y taladrar en odontología, y aplicaciones médicas en dermatología, } \\
\text { estomatologia y oftalmología (Becker, Olsson \& Simpson, 1999; Abramczyk, 2005). }\end{array}$} \\
\hline Tulio & \multicolumn{2}{|c|}{$\begin{array}{l}\text { Las fibras dopadas con tulio son atractivas para una variedad de aplicaciones en } \\
\text { espectroscopia, procesamiento de materiales y defensa (Babar et al., 2014). }\end{array}$} \\
\hline Itérbio & \multicolumn{2}{|c|}{$\begin{array}{l}\text { Fibras dopadas de láseres de alto poder en espectroscopia, procesamiento de mate- } \\
\text { riales y defensa (Engholm \& Norin, 2008; Babar et al., 2014). }\end{array}$} \\
\hline Lutecio & \multicolumn{2}{|c|}{ Scintillator para aplicaciones de espectroscopia, láseres (Bescher et al., 2000). } \\
\hline Itrio & \multicolumn{2}{|c|}{$\begin{array}{l}\text { Lámparas fluorescentes compactas, LED, televisores de pantalla delgada, aplicacio- } \\
\text { nes médicas, cerámicas (Hein et al., 2013). }\end{array}$} \\
\hline $\begin{array}{l}\text { Tierras raras } \\
\text { "livianas" }\end{array}$ & Tierras raras "pesadas" & \\
\hline
\end{tabular}


Tabla 4. Aplicaciones tecnológicas de materias primas críticas

\begin{tabular}{|c|c|}
\hline MPC & Aplicación tecnológica \\
\hline Magnesio & $\begin{array}{l}\text { Protección de corrosión, material de nanoestructuras, unión y fricción, solidificación, } \\
\text { compuestos con matriz de metal, nano compuestos (Hort, Mathaudhu, Ncclameg- } \\
\text { gham \& Alderman, 2013). }\end{array}$ \\
\hline Niobio & $\begin{array}{l}\text { Aumenta el endurecimiento del acero, superconductor en alta temperatura, vidrio } \\
\text { óptico, lentes de cámaras, condensadores electrónicos, superconductores magné- } \\
\text { ticos, componente de herramientas de corte, pantallas de dispositivos, aplicaciones } \\
\text { aeroespaciales (Alves \& Coutinho, 2015; Mishra \& Termsuksawad, 1999). }\end{array}$ \\
\hline Antimonio & $\begin{array}{l}\text { Baterías de bajo mantenimiento; compuesto alógeno retardante de fuego en plásticos, } \\
\text { tejidos, caucho; catálisis para productos PET, descolorante y refinación de agentes } \\
\text { para vidrio óptico y vidrio CRT, diodos, aleaciones de antimonio (baterías de ignición, } \\
\text { munición, bombas de tubos, revestimiento de tanques, tejas, soldadura, vainas de } \\
\text { cabos, rodamientos antifricción) fundición de plomo, pintura antirradioactiva, forro } \\
\text { de frenos de automóviles, catálisis, pesticidas, munición, medicina, detonadores, } \\
\text { marcadores de tanques (Halme et al., 2012). }\end{array}$ \\
\hline $\begin{array}{l}\text { Grafito } \\
\text { natural }\end{array}$ & $\begin{array}{l}\text { Aleaciones para frenos, fundición, lubricantes, aplicaciones refractarias, y siderurgia. } \\
\text { Baterías y procesamiento de aluminio (U.S. Geological Survey, 2015; Halme et al., } \\
\text { 2012) }\end{array}$ \\
\hline Tungsteno & $\begin{array}{l}\text { Metales duros, acero y otras aleaciones, otros productos (v.g., cabos, laminas y } \\
\text { varas, aplicaciones electrónicas, filamentos de luces incandescentes, tubos de vacío } \\
\text { y elementos de calentamiento, tubos de rayos X, interconexión de circuitos electró- } \\
\text { nicos, tubos de hornos siderúrgicos, sistemas de ignición de automóviles, electro- } \\
\text { dos de hornos de arco eléctrico). Aplicaciones químicas (v.g., porcelanas o catálisis, } \\
\text { fósforos y gel absorbente, inhibidor de corrosión, pigmentos e rodamientos) (British } \\
\text { Geological Survey, 2011). }\end{array}$ \\
\hline Germanio & $\begin{array}{l}\text { Fibra óptica, rayos infrarrojos, catálisis incolora para PET (tereftalo de polietileno), } \\
\text { industria electro solar, chips de silicio, circuitos integrados de alta velocidad, disposi- } \\
\text { tivos de comunicación Wireless, celdas fotovoltaicas de alta eficiencia para aplicacio- } \\
\text { nes espaciales, detectores de rayos gama, monocromators de rayos X, termo-foto- } \\
\text { voltaicos, aplicaciones para quimioterapia, y aleaciones (Melcher \& Buchholz, 2014). }\end{array}$ \\
\hline Indio & $\begin{array}{l}\text { Películas delgadas de pantallas de cristal líquido, compuestos de aleaciones, foto- } \\
\text { voltaicos, compuestos semiconductores en LEDs, soldaduras, polímeros ópticos, } \\
\text { enlaces de fusión, agentes de aleación, material de interface termal, baterías y mis- } \\
\text { celáneos (Schwarz-Schampera, 2014). }\end{array}$ \\
\hline Galio & $\begin{array}{l}\text { Celdas fotovoltaicas, LED, dispositivos optoelectrónicos, diodos láser, optoelectróni- } \\
\text { ca para aplicaciones espaciales, bienes de consumo, equipo industrial, equipo médi- } \\
\text { co, equipos de telecomunicaciones, aplicaciones de defensa, computadores de alto } \\
\text { desempeño, y equipos de telecomunicaciones (Zimmermann \& Gößling-Reisemann, } \\
\text { 2013; U.S. Geological Survey, 2015). }\end{array}$ \\
\hline
\end{tabular}


Materias primas críticas y complejidad económica en América Latina

Juan Sebastián Lara-Rodríguez - André Tosi Furtado - Aleix Altimiras-Martin

Continuación de la Tabla 4.

\begin{tabular}{|c|c|}
\hline $\begin{array}{l}\text { Silicio } \\
\text { metálico }\end{array}$ & $\begin{array}{l}\text { Pastillas oxidadas en la producción de circuitos integrados de supercomputadores, } \\
\text { fibra óptica de largas distancias y fibras para la creación de redes de telecomunica- } \\
\text { ciones (Platias, Vatalis \& Charalabidis, 2013). }\end{array}$ \\
\hline Cobalto & $\begin{array}{l}\text { Superaleaciones, principalmente de ramjets de aviones, aplicaciones de corte de alto } \\
\text { desgaste, varias otras aplicaciones metálicas, y una variedad de compuestos quími- } \\
\text { cos, baterías para autos eléctricos (U.S. Geological Survey, 2015; Busch et al., 2014) }\end{array}$ \\
\hline Fluorita & $\begin{array}{l}\text { Ácido fluorhídrico, fluoreto de aluminio, siderurgia, electrodos de arco eléctrico, es- } \\
\text { maltes, aislamiento de fibra de vidrio, vidrio y cemento portland, usos metalúrgicos, } \\
\text { alquilación de petróleo, reducción electrolítica y refinación de aluminio, iones de sodio } \\
\text { (Miller, 2010). }\end{array}$ \\
\hline Magnesita & $\begin{array}{l}\text { Productos refractarios para la industria siderúrgica, convertidores de oxígeno, hor- } \\
\text { nos eléctricos, cucharas de metalurgia secundaria (Csikósoya, Culkoya \& Antosoya, } \\
\text { 2013). }\end{array}$ \\
\hline Berilio & $\begin{array}{l}\text { Aleaciones aeroespaciales, discos duros y frenos; cerámicas de aislamiento de } \\
\text { electrónica de alta conductividad termal, aplicaciones de defensa (aviones militares, } \\
\text { vehículo espacial, sistemas de guía inerciales, electrónica de consumo, ventanas } \\
\text { de radar) reflectores en investigación de reactores nucleares y ventanas de rayos X } \\
\text { (Halme et al., 2012; McNeil, 2004). }\end{array}$ \\
\hline $\begin{array}{l}\text { Roca } \\
\text { fosfórica }\end{array}$ & $\begin{array}{l}\text { Ácido fosfórico, ácido superfosfórico, el cual es utilizado para la manufactura de } \\
\text { amonio líquido y granular, fertilizadores y suplementos alimenticios para animales, y } \\
\text { aditivos de comida y aplicaciones industriales (U.S. Geological Survey, 2015) }\end{array}$ \\
\hline $\begin{array}{l}\text { Carbón meta- } \\
\text { lúrgico }\end{array}$ & $\begin{array}{l}\text { El coque encuentra su aplicación principal en la industria del acero y el hierro como: } \\
\text { (1) reductor de óxidos del arrabio en el alto horno, (2) Material permeable para per- } \\
\text { mitir el fácil drenaje de hierro al fondo y llevar gases al tope, y (3) fuente de calor. } \\
\text { Gas, alquitrán, benzol bruto, licor amoniacal, y naftalina, larga cadena de compues- } \\
\text { tos alifáticos, y muchos oligoelementos como el germanio están presentes (Granda, } \\
\text { Blanco, Álvarez, Patrick \& Menéndez, 2014). }\end{array}$ \\
\hline Borato & $\begin{array}{l}\text { Ácido bórico, borato de sodio, ulexita (v.g., vidrios especiales y cerámicas), compo- } \\
\text { nentes abrasivos, productos de limpieza, insecticidas y producción de semiconduc- } \\
\text { tores (U.S. Geological Survey, 2015). }\end{array}$ \\
\hline Cromo & $\begin{array}{l}\text { Acero inoxidable, superaleaciones (v.g., paletas o láminas de motores de turbinas a } \\
\text { gas) (Gu, Harada \& Ro, 2004). }\end{array}$ \\
\hline Rutenio & $\begin{array}{l}\text { Modificador permanente para determinación de Al en plasma, enzima química en } \\
\text { biotecnología para reacciones de hidrogenación (Hoppstock y Sures, 2004). }\end{array}$ \\
\hline Rodio & $\begin{array}{l}\text { Catálisis en automóviles para reducir el óxido de nitrógeno, producción de vidrio de } \\
\text { alta calidad, cerámicas, circuitos de resistencia, y láminas conductoras (Hoppstock } \\
\text { \& Sures, 2004) }\end{array}$ \\
\hline
\end{tabular}


Continuación de la Tabla 4.

\begin{tabular}{|l|l|l|l|}
\hline Paladio & \multicolumn{2}{|l|}{$\begin{array}{l}\text { Aleaciones que contienen redes de cables, oxidación catalítica de diésel, modificador } \\
\text { químico, braquiterapia en cáncer de próstata, medicina dental (Hoppstock \& Sures, } \\
\text { 2004) }\end{array}$} \\
\hline Osmio & Colorante para tejido en microscopía electrónica (Hoppstock \& Sures, 2004) \\
\hline Iridio & Aleaciones especiales (Hoppstock \& Sures, 2004) \\
\hline Platino & $\begin{array}{l}\text { Aleaciones especiales, catálisis, reducción de óxidos de nitrógeno, oxidación catalíti- } \\
\text { ca de diésel, microanálisis de halogenuros, medicina dental, medicamentos antican- } \\
\text { cerígenos, joyas (Hoppstock \& Sures, 2004) }\end{array}$ \\
\hline & Metales del grupo de los platinoides (MGP) & & \\
\hline
\end{tabular}

GENERALIDADES SOBRE LA emergentes; (2) incremento de la comOFERTA DE MATERIAS PRI- petencia; (3) escalada del consumo de MAS CRÍTICAS

Desde la dinámica presentada, se espera que la demanda de metales tecnológicos se incremente considerablemente por las siguientes cuatro razones: (1) crecimiento de las economías recursos debido a la disminución de esperanza de vida del producto, como efecto del desgaste virtual de la actualización; y (4) falta de infraestructura de reciclaje para los metales tecnológicos (Köhler, Bakker \& Peck, 2013).

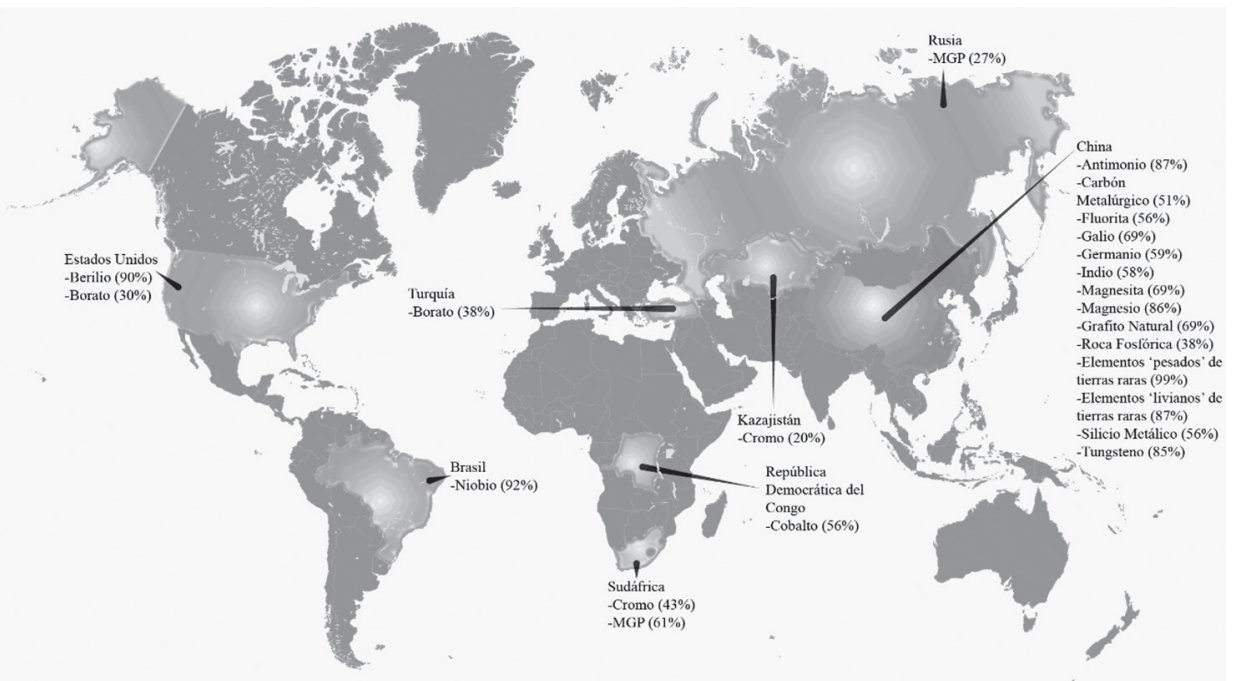

Figura 3. Principales países abastecedores de MPC para la UE.

Fuente: adaptación a partir de la European Commission (2014). 
Adicionalmente, en términos geopolíticos, Brown (2013) menciona que el principal productor del cobalto, la República Democrática del Congo, presenta inestabilidad política, exacerbada corrupción y un gobierno central deficiente. Paralelamente, en China hay problemas de gobernabilidad de un Estado de derecho, falta de rendición de cuentas, y la corrupción del Gobierno, lo que hace que no sea suficientemente capaz de afirmar un control de la producción. En algunos casos, como el mineral de tungsteno, China es el mayor importador y productor, lo que ha generado un mercado casi monopolizado. En la Figura 3 se indican en proporción los principales países productores de las MPC.

En el 2010, China reforzó sus cuotas de exportación de ETR llevando a los EUA, a la UE y a Japón a presentar el caso a la Organización Mundial de Comercio (OMC). Además, Wübbeke (2013) afirma que Beijing promueve un extenso conjunto de políticas, que incluyen reorganización industrial, conservación de recursos y protección ambiental, a fin de transformar los recursos naturales en instrumentos de poder político. Las principales reservas probadas de ETR se encuentran en la República Popular China, concentradas, en su orden, en las provincias de Mongolia Interior y la región sur de China, provincias de Juangxi, Guangdong, Fujian, Guanxi, Hunan y Yunnan.
Una situación próxima al monopolio de ciertas materias primas es, en primer lugar, el caso del niobio, que tiene como principal productor a Brasil. Para Alves y Coutinho (2015), este país mantiene el 98,53 \% de las reservas explotables del metal, representando aproximadamente el 97,2\% del total mundial. En este escenario, el estado de Minas Gerais es el mayor productor con un $83.6 \%$, seguido por el estado de Goiás con 15,3\% y el Amazonas con 1,1\%. En segundo lugar el berilio extraído en los EUA que, según el U.S. Geological Survey (2015), es producido en un $90 \%$ en este país, 7,5\% en China y 2,5\% en Mozambique, el $65 \%$ de las reservas probadas de este metal alcalino se encuentran en Gold Hill y Spor Mountain en el Estado de Utah y en la península de Seward estado de Alaska.

Como se indica en la Figura 3, para metales como el platino existe una mayor diversidad de oferentes, repartiendo su participación entre la Federación Rusa en la región de Krai de Krasnoyarsk y Sudáfrica en las provincias de Limpopo, Mpumangala y North West. El cromo, por otro lado, es producido igualmente en Sudáfrica como en Kazajistán, principalmente en la provincia de Aktyubinskn.

El proceso de reciclaje de estas materias primas críticas tiene por el momento una viabilidad relegada. Se puede argüir que los procesos de 
reutilización circulares para estos materiales se encuentran en una etapa naciente de aprendizaje, debido al intenso conocimiento científico y tecnológico requerido para separar un sinnúmero de insumos que componen los productos complejos (Altimiras-Martin, 2014). Es preciso mencionar que este punto requiere una atención especial por parte de los formuladores de política, ya que estos mercados deben ser prácticamente creados, partiendo de programas demostrativos, es decir, del lado de la oferta, junto con un equilibrado incentivo a la demanda por MPC recuperadas, o suscitadas de fuentes secundarias.

\section{Materias primas críticas en América Latina}

Las materias primas críticas han sido señaladas como primordiales para el funcionamiento de las economías complejas, por consiguiente de sistemas tecnológicos avanzados. Se catalogan de acuerdo con modelos teórico-conceptuales cambiantes según las demandas características de ciertos mercados. En otras palabras, esta diversa demanda radica en la complejidad económica de los países, debido a la acumulación e interacción de capacidades para agregar valor a ciertos minerales. Por tanto, se expande la hipótesis mostrada en la introducción en el sentido de que la variedad de materias primas críticas se justifica debido a la diversidad de producción de mercancías intensivas en conocimientos — representada en el ICE - como una consecuencia de la intensidad en I+D que impulsa a los respectivos sistemas nacionales de innovación. Relación que se refleja en un mejor desempeño de la producción de los países. En este sentido, se propone un ejercicio que se limitará a evidencias empíricas que tomarán en cuenta las políticas minerales formuladas para determinar estos materiales, con enfoque en las principales economías latinoamericanas, eludiendo implicaciones econométricas neoclásicas.

El PIB de América Latina —-20 países- aparece en la Tabla 5. Este fue calculado sin tomar en cuenta la producción de Cuba y Venezuela, por ausencia de los datos por parte del Banco Mundial para el periodo 2014. También se determinan las cinco mayores economías que representan el $87.3 \%$ de la producción regional y en las cuales se fijará el análisis. El promedio de intensidad en I+D de la tabla en mención se calculó para el periodo 2013, procesando la cifra 0 (cero) para Perú, Ecuador, República Dominicana, Costa Rica, Panamá, Bolivia, Honduras, Nicaragua, Haití y Venezuela, debido a su no procesamiento por parte del Banco Mundial. En cuanto al índice de complejidad económica (ICE), solo se soslayan las estimaciones de Haití por no ser evidentes en el Atlas de Complejidad Económica. 
Tabla 5. Producción, I+D y complejidad económica de América Latina

\begin{tabular}{|l|c|c|c|}
\hline & PIB en USD $^{\mathrm{a}}$ & I+ ${ }^{\mathrm{b}}$ & ICE $^{\mathrm{c}}$ \\
\hline América Latina & $5.593 .445 .896 .944,97$ & $0,30 \%$ & $-0,361$ \\
\hline Brasil & $2.416 .635 .506 .076,31$ & $1,15 \%$ & $-0,002$ \\
\hline México & $1.294 .689 .733 .233,03$ & $0,50 \%$ & 1,041 \\
\hline Argentina & $537.659 .972 .702,09$ & $0,58 \%$ & $-0,219$ \\
\hline Colombia & $377.739 .622 .865,84$ & $0,23 \%$ & $-0,187$ \\
\hline Chile & $258.061 .522 .886,53$ & $0,36 \%$ & $-0,360$ \\
\hline
\end{tabular}

Notas: a(The World Bank, 2014) ${ }^{b}$ (The World Bank, 2013) c(Hausmann et al., 2014).

Las principales economías de América Latina protagonizan un papel moderado con la extracción de 37 materias primas no energéticas en el escenario global (Figura 4). Brasil es líder regional en producción de 22 minerales, con una participación excepcional en la oferta de niobio, y considerable en bauxita, hierro, tantalio, talco, metal de magnesio, níquel y grafito; le sigue México con la producción de 17 minerales, entre los que se destacan la plata, fluorita, bismuto, estroncio, wollastonita y el zinc, en cantidades relevantes; Chile es el tercer mayor productor de tipos de minerales de los países seleccionados, con 13 materias primas, en volúmenes relevantes para el caso del yodo, renio, litio, cobre, molibdeno y boro; Argentina extrae seis materiales con un volumen notable para el metaloide boro; Colombia, por último, tiene una ligera participación en la producción de níquel en el mercado global.

Ahora bien, se ha determinado que el portafolio de materias primas minerales no energéticas en América Latina se focaliza en minerales que no son denominados como críticos por la UE o los EUA. Sin embargo, sobresalen unas excepciones como la de Brasil, pues cuenta con reservas probadas de niobio, magnesio, grafito, silicio y roca fosfórica; México, con fluorita; Chile, con cobre, litio, molibdeno y boro; y la Argentina, con depósitos de boro y litio. 
Apuntes CENES Volumen 37, Número 65

enero - junio 2018. Págs. 15-51

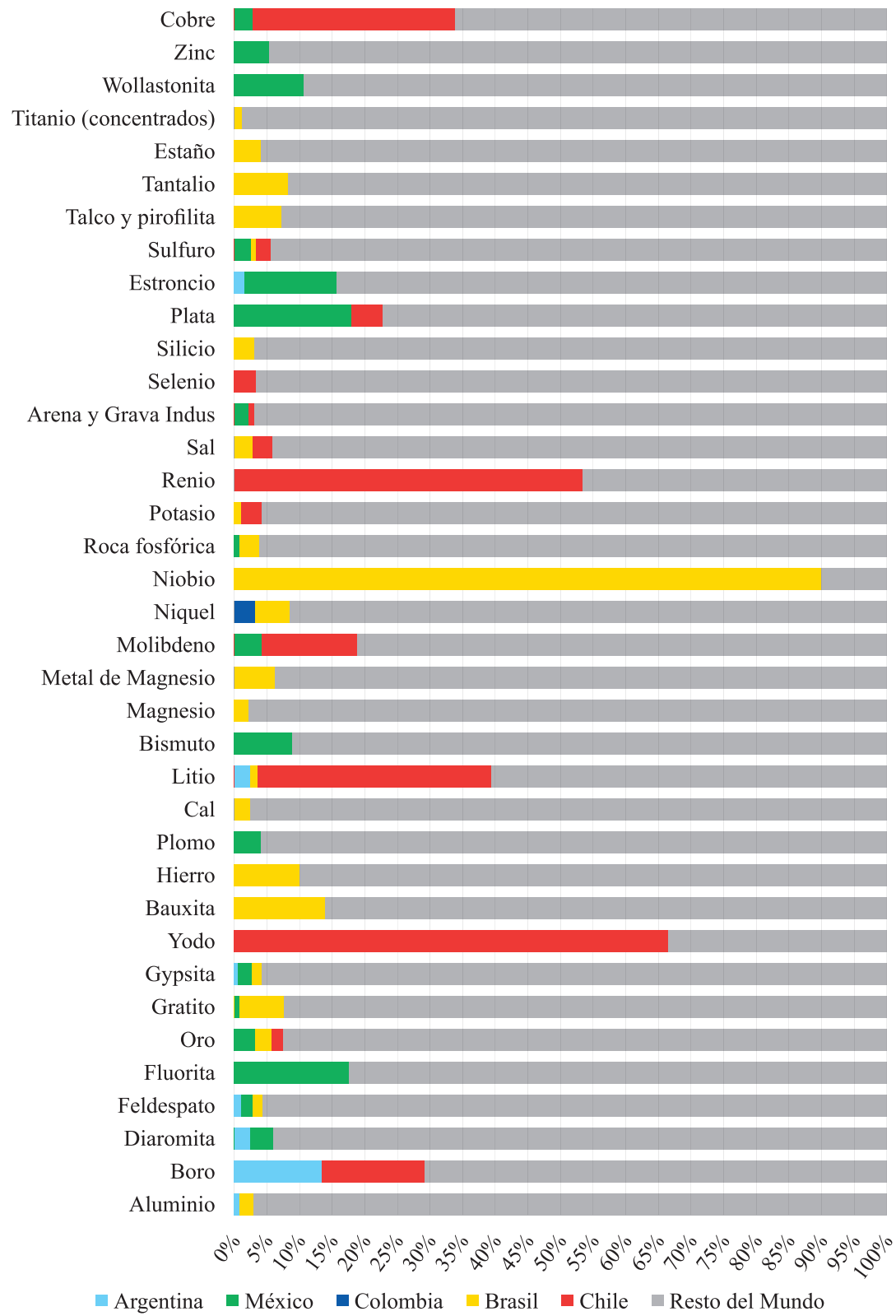

Figura 4. Principales economías de América Latina y su producción mineral en el mercado mundial.

Fuente: adaptación a partir el U.S. Geological Survey (2015). 
En Brasil es el Ministério de Minas e Energia (MME) el encargado de formular la política mineral del país, y el Departamento Nacional de Produção Mineral, parte del MME, es el que administra los recursos minerales de ese país. Las directrices generales para la geología, recursos minerales, minería, transformación mineral y metalurgia, se determinan en el "Plano Nacional de Mineração 2030" (Ministério de Minas e Energia, 2011). En este se hace referencia a mineral estratégico en tres situaciones:

(1) De bienes minerales, de los cuales Brasil depende de su importación en un alto porcentaje para el abastecimiento de los siguientes sectores vitales para su economía: el sector agrícola, con una dependencia en un $90 \%$ de importaciones para el potasio, $70 \%$ para el nitrógeno y $50 \%$ para el fósforo; y el sector siderúrgico integrado que requiere un $100 \%$ de importaciones de carbón metalúrgico o coque; (2) minerales que deberán crecer en importancia en las próximas décadas por su aplicación en productos de alta tecnología, entre estos los ETR, litio, cobalto, tantalio y otros denominados "portadores de futuro"; y (3) minerales esenciales para la economía por su generación de divisas, principalmente el hierro y el niobio.

Para México, la Secretaría de Economía es la que formula y evalúa la política del sector minero, la cual es implementada desde la Coordinación General de Minería. El "Programa de Desarrollo Minero 2013-2018" (Secretaría de Economía, 2014) señala las metas, estrategias y acciones para el sector en este periodo. No establece categóricamente minerales estratégicos o críticos, materias primas críticas, metales tecnológicos, etc., sino que presenta una breve semblanza en la estrategia "1.2. Promover la diversificación en la explotación y aprovechamiento de minerales, favoreciendo los de interés industrial" (p. 30). Se infiere que le corresponde aumentar acciones de exploración al Servicio Geológico Mexicano para la determinación de reservas de hierro y tierras raras.

El Ministerio de Minería es el encargado en Chile de desarrollar la política pública mineral, desde organismos como la Corporación Nacional del Cobre, el Servicio Nacional de Geología y Minería, la Comisión Chilena del Cobre y la Empresa Nacional de Minería. En lo relacionado con la clasificación de minerales críticos, la Comisión Chilena del Cobre (2014) identifica una serie de insumos críticos para el desarrollo de la minería en este país, y desarrolla una metodología que intenta adaptar el concepto expuesto en los modelos de materias primas críticas de la UE y los EUA. Determina los insumos críticos para la industria del cobre en gastos de capital (v.g., camiones, pala de cable, molinos, geomembrana, correas 
transportadoras) y gastos operacionales (v.g., energía eléctrica, agua fresca, combustibles, ácido sulfúrico, servicios de mantenimiento y reparación). Por otro lado, establece que la creación de la Comisión Nacional del Litio (Ministerio de Minería, 2015) busca la formulación de una política mineral sostenible para este metal.

En la Argentina, la formulación de políticas minerales se encuentra a cargo del Ministerio de Energía y Minería. El sector es regulado mediante la Ley n. 1919 o Código de Minería (República Argentina, 1887), que en su artículo 354 establece que el poder ejecutivo nacional, con los ministerios de Defensa y de Economía, en coordinación con las Fuerzas Armadas, clasificará periódicamente las sustancias minerales estratégicas, para los fines señalados en el Código. No obstante, como hace saber la Secretaría de Política Económica y Planificación del Desarrollo (2016), se identifica como uno de los principales desafíos del sector minero en este país, la identificación y planificación de la explotación de minerales estratégicos.

En Colombia, la política pública mineral está a cargo del Ministerio de Minas y Energía (2012), el cual determinó unos minerales de interés estratégico para el país, basado en estudios del Servicio Geológico Colombiano, en donde se establece un potencial para el hallazgo de oro, metales del grupo platino, cobre, hierro, coltán, fosfato de potasio, magnesio, uranio y carbón metalúrgico. Además afirma que los minerales estratégicos son fundamentales para el desarrollo minero del país y la diversificación de su canasta minera. Simultáneamente, la entidad adscrita al Ministerio, la Unidad de Planeación Minero Energética (2013), en el plan de desarrollo minero a 2014, menciona los tres atributos de mineral estratégico, expuestos anteriormente por el "Plano Nacional de Mineração 2030" de Brasil.

\section{DISCUSIÓN}

Para el caso de Brasil, (1) la determinación de los minerales estratégicos carece de un modelo metodológico cualitativo y cuantitativo, que tome en cuenta múltiples variables que puedan determinar irrupciones en el abastecimiento de los minerales de la primera situación; (2) se evidencia una débil intención para determinar otros materiales claves para sectores promisorios de su economía (v.g., aeronáutico, aeroespacial, optoelectrónico y automovilístico) aparte del siderúrgico y agrícola; (3) en la segunda situación no se identifican sistemas tecnológicos para ser desarrollados y se parte de la noción de la disponibilidad de ETR en un volumen de reservas que no se encuentran en etapa de explotación; (4) es nebuloso el panorama para el desarrollo de cadenas de agregación 
de valor para las MPC como el niobio, magnesio, grafito, silicio y roca fosfórica, en donde principalmente se podrían impulsar industrias que generen productos complejos. Y por último, (5) existió una escasa participación del entonces Ministério de Ciência e Tecnologia, o de la Companhia de Pesquisa em Recursos Minerais, como agentes del SNI en la determinación de los materiales estratégicos.

En Brasil resulta paradójica la moderada inversión en $\mathrm{I}+\mathrm{D}$ y la exigua complejidad de su economía, la cual se concentra en el sector agropecuario y mineral. Esta se puede entender por el poco involucramiento de actividades de ciencia y tecnología en la industria nacional, y la débil eficacia en la convergencia a nuevos productos intensivos en conocimiento. Parece que la investigación y el desarrollo se dirigen más a la producción de conocimiento científico, antes que a la invención de productos y procesos para ser difundidos en el mercado, lo que posibilitaría innovaciones en torno a la cadena de sus considerables recursos minerales probados.

En el caso de México se logra evidenciar que la política mineral está orientada a fortalecer la oferta de los minerales actualmente extraídos y a afianzar el plausible programa $\mathrm{Fi}$ deicomiso de Fomento Minero (FOMANI) como institución de fomento especializada en la minería a pequeña y mediana escala, concomitante con la intensificación de la exploración de nuevas áreas. No obstante, se elucida la falta de determinación de los minerales estratégicos para su industria, por ejemplo, no son catalogados para la siderúrgica y la electrónica, por cuanto para la primera se requiere hierro y manganeso, y para la segunda, de silicio barita y caolín, que son en su mayoría importados. Se mencionan minerales de interés industrial, pero no los cataloga o da visos de la creación de un modelo metodológico que los capture. Igualmente se dejan de lado otros sectores industriales emergentes, obviando una nivelación en intensidad de $\mathrm{I}+\mathrm{D}$ al de economías complejas, como la cooperación activa del Consejo Nacional de Ciencia y Tecnología (Conacyt) para la definición de MPC para la economía mexicana.

Una explicación de la ligereza en la formulación de la política mineral en MPC para su propia industria, y de la destacable complejidad económica de México en contraste con la presente en América Latina, se basa en que las casas matrices de las empresas filiales, fabricantes de los productos complejos y cuyas plantas se localizan principalmente en los Estados fronterizos con los EUA, se hacen a cargo de la estabilidad en el abastecimiento de sus materias primas. Es preciso mencionar que la industria maquiladora de exportación es prevaleciente en el aparato productivo mexicano, 
como resultado de una precoz implantación de una industrialización en promoción de exportaciones y de su cercanía geográfica con los robustos mercados de América del Norte.

En Chile es evidente una marcada dependencia económica de las industrias extractivas. El cobre, por ejemplo, representa aproximadamente el $10 \%$ de su PIB, este rasgo hace que su economía sea la más simple de los países de América Latina tratados, evidenciando una diversificación productiva endeble. Las MPC para sus sectores económicos no son establecidas oficialmente, y se nota un interés por continuar concentrando sus operaciones en el sector cuprífero, como es consignado en la hoja de ruta tecnológica del sector, contenida en el Programa Nacional de Minería Alta Ley (2016), en la cual se busca potenciar la minería del cobre como plataforma para la generación de innovación tecnológica. En este programa, sorpresivamente, no se determinan actores, medios y acciones para el establecimiento de programas de ciencia y tecnología que busquen la interacción del litio, molibdeno y boro de los cuales el país dispone. Por otro lado, si bien las comisiones tanto del cobre como del litio corresponden a procesos de formulación de política pública razonables, urge una mayor participación de la Comisión Nacional de Investigación Científica y Tecnológica (Conicyt), y multiplicar la inversión en $\mathrm{I}+\mathrm{D}$ para convertir esos mine- rales en productos complejos.

Para Argentina, la caracterización de estos minerales es aún indeterminada. Su economía presenta trazos de simpleza, dada la hegemonía del sector agropecuario, no obstante con una industria automotriz de relativa importancia. La política mineral se dirige principalmente al impulso de la exploración en áreas argentino-chilenas con potencial de tenores en cobre, plata y oro, además busca facilitar la inversión extranjera directa en el sector mediante subvenciones fiscales. Por lo tanto, es conveniente adicionar las MPC a su industria siguiendo criterios de modelaje conceptual válidos y en sintonía con el Ministerio de Ciencia, Tecnología e Innovación Productiva. En este sentido, el SNI de este país cuenta con una estructura prometedora, que requiere ser exponencialmente robustecida en intensidad de $\mathrm{I}+\mathrm{D}$, para condensar conocimiento dirigido a agregar valor a elementos existentes como el boro y el litio.

Entre los países analizados, Colombia es la nación que presenta menor desarrollo del sector minero. Su política mineral se concentra principalmente en impulsar la prospectiva geológica de áreas, en sincronía con la formalización del sector, que en gran parte es desempeñado por organizaciones artesanales y de pequeña escala (La- 
ra-Rodríguez et al., 2017). La complejidad de la economía es limitada y muestra atisbos de dependencia de la exportación de petróleo y carbón térmico. Prueba de la situación es la determinación de minerales estratégicos fundada en un potencial que se basa en reservas hipotéticas y carece de un modelo razonable que determine las MPC para su propia industria. Otro agravante es la efímera intensidad en I+D del país, que se encuentra por debajo del promedio de América Latina, poniendo en evidencia la fabricación espuria de productos complejos. Estas conjeturas predisponen un sinuoso camino para afianzar un SNI eficiente por parte del Departamento Administrativo de Ciencia, Tecnología e Innovación (Colciencias), que busque la creación de capacidades para agregar valor a los recursos minerales optimistamente previstos, más aun ante un escenario de pacificación.

\section{CONCLUSIONES}

Se constata la hipótesis prima del estudio, según la cual, la complejidad económica de una nación se establece por la variedad de sus materias primas críticas. Se argumenta que la Unión Europea determina 20 materias primas críticas; los EUA, 13 minerales críticos, y en el proceso se vinculan directamente a sus respectivos sistemas nacionales de innovación. Estas economías cuentan con un alto índice de complejidad económica y una considerable intensidad en I+D. En cuanto a América Latina, sus principales economías son simples y de irrisoria intensidad en $\mathrm{I}+\mathrm{D}$, siendo la determinación de materias primas críticas prácticamente inexistente, así como la vinculación del sistema nacional de innovación a la política mineral. Consecuentemente, se arguye que para mantener un ICE significativo, se debe contar con una intensidad en $\mathrm{I}+\mathrm{D}$ equivalente y considerable, que permita la dinamización de los sistemas naciones de innovación dirigidos a la producción y el desarrollo de productos complejos.

En general, a partir del análisis realizado enfocado en la política mineral de materias primas críticas, es urgente que América Latina comience a generar una agenda política y económica dirigida a impulsar sus respectivos SNI. De lo contrario, se continuarán ahondando esas brechas de complejidad económica que hacen que en la región sus economías sean inusitadamente simples.

Para finalizar, es necesario enfatizar que algunos de los productos complejos producidos con las MPC son de enorme importancia para disminuir las externalidades negativas asociadas a actividades antrópicas, señaladas en la introducción de este artícu- 
1o. En este aspecto, América Latina se encuentra rezagada, no solamente por no desarrollar estos productos intensivos en conocimiento, sino también por instaurar una dependencia tecnológica de las naciones que desarrollan las denominadas tecnologías verdes.

\section{AGRADECIMIENTOS}

Este artículo es un producto asociado al proceso n. ${ }^{\circ}$ 131999/2015-0 del Con- selho Nacional de Desenvolvimento Científico e Tecnológico (CNPq) de Brasil y al proyecto "El desarrollo sostenible de la minería del platino en Colombia" http://doi.org/10.13140/ RG.2.2.15227.92963. Los autores agradecemos los comentarios de los árbitros anónimos, que ayudaron a mejorar el manuscrito. Igualmente, a los investigadores Lorenz Erdmann y Kalle Artturi Piirainen por sus observaciones. 


\section{REFERENCIAS}

Abramczyk, H. (2005). Introduction to Laser Spectroscopy (First). Amsterdam: Elsevier B.V. http://doi.org/10.1016/B978-044451662-6/50014-9

Altimiras-Martin, A. (2014). Analysing the Structure of the Economy Using Physical Input-Output Tables. Economic Systems Research, 26(4), 463485. http://doi.org/10.1080/09535314.2014.950637

Alves, A. R., \& Coutinho, A. dos R. (2015). The Evolution of the Niobium Production in Brazil. Materials Research, 18(1), 106-112. http://doi. org/10.1590/1516-1439.276414

Auty, R. M. (2003). Natural resources, development models and sustainable development. In International Institute for Environment and Development, Environmental Economics Programe (pp. 0-25). Stevenage, UK: Earthprint Limited. Retrieved from http://eprints.lancs.ac.uk/9356/

Auty, R. M. (2007). The resources curse and sustainable development. In G. Atkinson, S. Dietz, \& E. Neumayer (Eds.), Handbook of Sustainable Development (Vol. I, pp. 207-219). Cheltenham, UK and Northampton, MA, USA: Edward Elgar Publishing.

Babar, I. M., Sabran, M. B. S., Jusoh, Z., Ahmad, H., Harun, S. W., Halder, A., Bhadra, S. K. (2014). Double-clad thulium/ytterbium co-doped octagonal-shaped fibre for fibre laser applications 1. Ukrainian Journal of Physical Optics, 15(4), 173-184.

Becker, P. C., Olsson, N. A., \& Simpson, J. R. (1999). Introduction. In Erbium-Doped Fiber Amplifiers (First, pp. 1-11). London, GBR: Academic Press. http://doi.org/10.1016/B978-012084590-3/50003-X

Bescher, E., Robson, S. R., Mackenzie, J. D., Patt, B., Iwanczyk, J., \& Hoffman, E. J. (2000). New lutetium silicate scintillators. Journal of Sol-Gel Science and Technology, 19(3), 325-328. http://doi.org/10.1023/A:1008785616233

British Geological Survey. (2011). Tungsten profile. Nottingham. Retrieved from www.MineralsUK.com 
Brown, A. (2013). By the numbers: critical materials--weak spot for the U.S.? Mechanical Engineering [Serial Online], 135(5), 28-29. Retrieved from Business Source Complete, Ipswich, MA. Accessed July 2, 2014.

Brumme, A. (2014). Wind Energy Deployment and the Relevance of Rare Earths - An Economic Analysis. In Wind Energy Deployment and the Relevance of Rare Earths, An Economic Analysis (1st ed.). Berlin: Springer Fachmedien Wiesbaden. http://doi.org/10.1007/978-3-658-04913-3

Busch, J., Steinberger, J. K., Dawson, D. a, Purnell, P., \& Roelich, K. (2014). Managing critical materials with a technology-specific stocks and flows model. Environmental Science \& Technology, 48(2), 1298-305. http://doi. org/10.1021/es404877u

Chakhmouradian, A. R., Smith, M. P., \& Kynicky, J. (2015). From "strategic" tungsten to "green" neodymium: A century of critical metals at a glance. Ore Geology Reviews, 64, 455-458. http://doi.org/10.1016/j.oregeorev.2014.06.008

Comisión Chilena del Cobre. (2014). Identificación de insumos críticos para el desarrollo de la minería en Chile. Santiago de Chile. Retrieved from http:// www.cochilco.cl/descargas/estudios/informes/Insumos Críticos/Estudio_ de_Insumos_Criticos_en_la_Mineria_Chilena_VF.pdf

Csikósoya, A., Ćulkoya, K., \& Antośoya, M. (2013). Magnesite industry in the Slovak Republic. Gospodarka Surowcami Mineralnymi - Mineral Resources Management, 29(3). http://doi.org/10.2478/gospo-2013-0028

Dosi, G. (1982). Technological paradigsm and tecnological trajectories. Research Policy, 11, 147-162. http://doi.org/https://doi.org/10.1016/00487333(82)90016-6

Du, X., \& Graedel, T. E. (2013). Uncovering the end uses of the rare earth elements. The Science of the Total Environment, 461-462, 781-4. http://doi. org/10.1016/j.scitotenv.2013.02.099

Engholm, M., \& Norin, L. (2008). Preventing photodarkening in ytterbium-doped high power fiber lasers; correlation to the UV-transparency of the core glass. Optics Express, 16, 1260-1268. http://doi.org/10.1364/OE.16.001260 
Erdmann, L., \& Graedel, T. E. (2011). Criticality of non-fuel minerals: A review of major approaches and analyses. Environmental Science and Technology, 45, 7620-7630. http://doi.org/10.1021/es200563g

European Commission. (2014). Report on critical raw materials for the EU, Report of the Ad hoc Working Group on defining critical raw materials. Brussels. Retrieved from http://ec.europa.eu/enterprise/policies/raw-materials/ files/docs/crm-report-on-critical-raw-materials_en.pdf

Fromer, N. a., \& Diallo, M. S. (2013). Nanotechnology and clean energy: sustainable utilization and supply of critical materials. Journal of Nanoparticle Research, 15(11), 1-15. http://doi.org/10.1007/s11051-013-2011-9

Glöser, S., Tercero, L., Gandenberger, C., \& Faulstich, M. (2015). Raw material criticality in the context of classical risk assessment. Resources Policy, 44, $35-46$.

Goe, M., \& Gaustad, G. (2014). Identifying critical materials for photovoltaics in the US: A multi-metric approach. Applied Energy, 123, 387-396. http:// doi.org/10.1016/j.apenergy.2014.01.025

Goonan, T. (2011). Rare Earth Elements - End Use and Recyclability. Reston, Virginia: U.S. Geological Survey Scientific Investigations Report 20115094. Retrieved from http://pubs.usgs.gov/sir/2011/5094/

Graedel, T. E., Barr, R., Chandler, C., Chase, T., Choi, J., Christoffersen, L., ... Zhu, C. (2012). Methodology of metal criticality determination. Environmental Science and Technology, 46(2), 1063-1070. http://doi.org/10.1021/ es $203534 z$

Granda, M., Blanco, C., Alvarez, P., Patrick, J. W., \& Menéndez, R. (2014). Chemicals from coal coking. Chemical Reviews, 114(3), 1608-1636. http://doi.org/10.1021/cr400256y

Gu, Y. F., Harada, H., \& Ro, Y. (2004). Chromium and chromium-based alloys: Problems and possibilities for high-temperature service. Jom, 56(9), 28 33. http://doi.org/10.1007/s11837-004-0197-0

Gupta, V. K., Jain, R., Hamdan, a. J., Agarwal, S., \& Bharti, A. K. (2010). A novel ion selective sensor for promethium determination. Analytica Chimica Acta, 681(1-2), 27-32. http://doi.org/10.1016/j.aca.2010.09.037 
Halme, K., Piirainen, K., Vekinis, G., Ernst-Udo, S., \& Viljamaa, K. (2012). Substitutionability of Critical Raw Materials. Zhurnal Eksperimental'noi i Teoreticheskoi Fiziki. Brussels: European Union. http://doi.org/10.2861/53633

Hartwick, J. M. (1977). Intergenerational Equity and the Investing of Rents from Exhaustible Resources. American Economic Association, 67(5), 972-974. Retrieved from http://www.jstor.org/stable/1828079

Hausmann, R., Hidalgo, C. a., Bustos, S., Coscia, M., Chung, S., Jimenez, J., ... Yildirim, M. (2014). The Atlas of Economic Complexity: Mapping Paths to Prosperity (2014th ed.). Cambridge, MA, USA: Harvard University and Masachussetts Institute of Technology. Retrieved from http://atlas.cid.harvard.edu/rankings/

Hein, J. R., Mizell, K., Koschinsky, A., \& Conrad, T. a. (2013). Deep-ocean mineral deposits as a source of critical metals for high- and green-technology applications: Comparison with land-based resources. Ore Geology Reviews, 51, 1-14. http://doi.org/10.1016/j.oregeorev.2012.12.001

Hensel, N. D. (2011). Economic Challenges in the Clean Energy Supply Chain: The Market for Rare Earth Minerals and Other Critical Inputs. Business Economics, 46(3), 171-184. http://doi.org/10.1057/be.2011.17

Hidalgo, C. a, \& Hausmann, R. (2009). The building blocks of economic complexity. Proceedings of the National Academy of Sciences of the United States of America, 106(26), 10570-10575. http://doi.org/10.1073/ pnas.0900943106

Hoppstock, K., \& Sures, B. (2004). Platinum-Group Metals. In E. Merian, M. Anke, \& M. Stoeppler (Eds.), Elements and Their Compounds in the Environment: Occurrence, Analysis and Biological Relevance (pp. 1047-1086). Weinheim, Germany: WILEY-VCH Verlag GmbH\&Co. KGaA. http://doi. org/10.1002/9783527619634.ch41

Hort, N., Mathaudhu, S., Ncclameggham, N., \& Alderman, M. (2013). Magnesium Technology 2013. (M. \& M. S. (TMS) Magnesium Committee of the Light Metals Division of The Minerals, Ed.). San Antonio: Wiley. 
Karl, T. L. (1997). Review The Paradox of Plenty: Oil Booms and Petro-States. Berkeley: University of California Press.

Köhler, A. R., Bakker, C., \& Peck, D. (2013). Critical materials: a reason for sustainable education of industrial designers and engineers. European Journal of Engineering Education, 38(4), 441-451. http://doi.org/10.1080/030437 97.2013.796341

Lara-Rodríguez, J. S., \& Bermúdez, J. I. (2011). Perspectiva de la política de innovación y su monitoreo en la Unión Europea , 2010-2020. Finanzas Y Política Económica, 3(2), 105-126. Retrieved from http://ideas.repec. org/a/col/000443/009853.html

Lara-Rodríguez, J. S., Rojas, C. A., \& Martínez, J. A. (2015). Evolución organizacional : inducción socio-biológica para el entendimiento de la metáfora. AD-Minister, 26(enero-junio), 101-122. http://doi.org/10.17230/ad-minister.26.5

Lara-Rodríguez, J. S., Naranjo-Merchán, W., \& Manosalva, S. R. (2017). Formación de capacidades para la formalización minera en Colombia: Un estudio de investigación acción. Cuadernos Del CENDES, 34(94), 97-126. Extraído de http://www.redalyc.org/pdf/403/40353171006.pdf

Lundvall, B. Å., Vang, J., Chaminade, J., \& Chaminade, C. (2009). Innovation system research and developing countries. In B. A. Lundvall, K. J. Joseph, C. Chaminade, \& J. Vang (Eds.), Handbook of Innovation Systems and Developing Countries, Building Domestic Capabilities in a Global Setting (pp. 1-30). Cheltenham, UK and Northampton, MA, USA: Edward Elgar Publishing.

Massari, S., \& Ruberti, M. (2013). Rare earth elements as critical raw materials: Focus on international markets and future strategies. Resources Policy, 38(1), 36-43. http://doi.org/10.1016/j.resourpol.2012.07.001

McNeil, D. (2004). Beryllium. London, GBR. Retrieved from http://beryllium. eu/resources/Critical Material and Market Forces Literature/Beryllium Production and Outlook Roskill Mineral Sevices.pdf 
Melcher, F., \& Buchholz, P. (2014). Germanium. In G. Gunn (Ed.), Critical Metals Handbook (First, pp. 177-203). Nottingham. UK: John Wiley \& Sons. http://doi.org/10.1002/9781118755341.ch8

Miller, M. (2010). Fluorspar. Mining Engineering, 62(6), 48-49. Retrieved from http://search.proquest.com/docview/578164423? accountid=8113

Ministério de Minas e Energia. (2011). Plano Nacional de Mineração 2030. Geologia, Mineração e Transformação Mineral. Brasilia. Retrieved from http:// www.mme.gov.br/documents/1138775/1732821/Book_PNM_2030_2. pdf/f7cc76c1-2d3b-4490-9d45-d725801c3522

Ministerio de Minas y Energía. (2012). Resolución número 180102 de 30 enero de 2012 "Por la cual se determinan unos minerales de interés estratégico para el país." Bogotá D.C.: República de Colombia. Retrieved from http:// www.minminas.gov.co/documents/10180//23517//20337-10498.pdf

Ministerio de Minería. (2015). Ministerio de Minería - Cuenta Pública. Santiago de Chile. Retrieved from http://www.gob.cl/cuenta-publica/2015/sectorial/2015_sectorial_ministerio-mineria.pdf

Mishra, B., \& Termsuksawad, P. (1999). Niobium. Review of Extraction, Processing, Propierties and Aplications of Reactive Metals, 83-134. http://doi. org/DOI: 10.1002/9781118788417.ch3

National Research Council of the National Academies. (2008). Minerals, critical minerals, and the U. S. economy. Washington, D.C.: National Academies Press : Washington, DC, United States. Retrieved from www.nap.edu

Nelson, R. R., \& Winter, S. G. (1982). An evolutionary Theory of Economic Change. Cambridge, MA, USA: Harvard University Press.

Platias, S., Vatalis, K. I., \& Charalabidis, G. (2013). Innovative Processing Techniques for the Production of a Critical Raw Material the High Purity Quartz. Procedia Economics and Finance, 5(13), 597-604. http://doi.org/10.1016/ S2212-5671(13)00070-1

Ploeg, F. Van Der. (2011). Natural Resources: Curse or Blessing? Journal of Economic Literature, 49(2), 366-420. http://doi.org/10.1257/jel.49.2.366 
Programa Nacional de Minería Alta Ley. (2016). Desde el cobre a la innovación. Roadmap Tecnológico 2015-2035. (Fundación Chile, Ed.). Santiago de Chile: A Impresores.

República Argentina. (1887). Ley 1919 Código de Minería. Buenos Aires: Senado y Camara de Diputados. Retrieved from http://wp.cedha.net/ wp-content/uploads/2011/10/ley-minera-argentina.pdf

Schwarz-Schampera, U. (2014). Indium. In G. Gunn (Ed.), Critical Metals handbook (First, Vol. 11, pp. 204-229). Nottingham. UK: John Wiley \& Sons. http://doi.org/10.1002/9781118755341.ch9

Secretaría de Economía. (2014). Programa de Desarrollo Minero 2013-2018. Ciudad de México. Retrieved from http://www.dof.gob.mx/nota_detalle. php? codigo $=5344070 \&$ amp; fecha $=09 / 0$

Secretaría de Política Económica y Planificación del Desarrollo. (2016). Informes de cadenas de valor: Minería Metalífera y Rocas de Aplicación. Buenos Aires. Retrieved from http://www.economia.gob.ar/peconomica/ docs/ficha_litio_dic_2011.pdf

Senate Committee on Interior and Insular Affairs. (1954). Accessibility of strategic and critical materials to U.S. in time of war and for expanding economy. Accessibility of Strategic and Critical Materials to the United States in Time of War and for Our Expanding Economy. Report of the Committee on Interior and Insular Affairs Made by Its Minerals, Materials, and Fuels Economic Subcommittee pursuant to S. Re. Retrieved from http:// ezproxy.unal.edu.co/login?url=http://search.ebscohost.com/login.aspx?direct=true \&db=edslns\&AN=LNSD80B819B-90F7F8E3\&lang=es\&site=e ds-live

Sievers, H., Buijs, B., \& Tercero Espinoza, L. a. (2012). Limits to the critical raw materials approach. Proceedings of the ICE - Waste and Resource Management, 165(4), 201-208. http://doi.org/10.1680/warm.12.00010

Slowinski, G., Latimer, D., \& Mehlman, S. (2013). Research-on-Research: Dealing with Shortages of Critical Materials. Research-Technology Management, 56(5), 18-24. http://doi.org/10.5437/08956308X5605139 
The World Bank. (2013). World Development Indicators: Science and technology. Washington, DC, USA: World Bank Group. Retrieved from http://wdi. worldbank.org/table/5.13

The World Bank. (2014). World Bank GDP Deflator. Retrieved from http://data. worldbank.org/indicator/NY.GDP.DEFL.KD.ZG)

U.S. Geological Survey. (2015). Mineral Commodity Summaries 2015. Reston, Virginia. Retrieved from http://minerals.usgs.gov/minerals/pubs/ mcs/2015/mcs2015.pdf

Unidad de Planeación Minero Energética. (2013). Plan Nacional De Desarrollo Minero 2010 - 2014. Bogotá D.C. Retrieved from http://www.upme.gov. co/Docs/pndm/2013/PNDM2014.pdf

Van Gosen, B., Verplanck, P., Long, K., Gambogi, J., Joseph, \& Seal. (2014). The Rare-Earth Elements - Vital to Modern Technologies and Lifestyles. U.S. Geological Survey Fact Sheet 2014-3078. Reston, Virginia: U.S. Geological Survey Fact Sheet 2014-3078. http://doi.org/http://dx.doi.org/10.3133/ fs20143078

World Commission on Environment and Development. (1987). Report of the World Commission on Environment and Development: Our Common Future (The Brundtland Report). Medicine, Conflict and Survival. http://doi. org/10.1080/07488008808408783

Wübbeke, J. (2013). Rare earth elements in China: Policies and narratives of reinventing an industry. Resources Policy, 38(3), 1-11. http://doi.org/10.1016/j. resourpol.2013.05.005

Ziemann, S., Grunwald, A., Schebek, L., Müller, D. b., \& Weil, M. (2013). The future of mobility and its critical raw materials. Revue de Métallurgie, 110(1), 47-54. http://doi.org/10.1051/metal/2013052

Zimmermann, T., \& Gößling-Reisemann, S. (2013). Critical materials and dissipative losses: a screening study. The Science of the Total Environment, 461-462, 774-80. http://doi.org/10.1016/j.scitotenv.2013.05.040 